INVITED REVIEW

\title{
Gastrointestinal Motility Disorders in Critically Ill
}

\author{
Deepak Govil ${ }^{1}$, Divya Pal ${ }^{2}$
}

\begin{abstract}
Gastrointestinal (GI) motility disorders are a common problem in the intensive care unit (ICU) and are associated with increased morbidity, complications like feeding intolerance, malnutrition, bacterial translocation, sepsis, multiple organ failure, prolonged hospital stay and increased risk of mortality. Several risk factors in intensive care setting that contribute to Gl dysmotility include mechanical ventilation, vasopressor support, use of opioids, etc. Degree of GI motility dysfunction correlates with severity of critical illness. So, it is important to identify the dysfunction early so that targeted therapy could be prescribed to prevent worsening of clinical outcomes.

Keywords: Gastroparesis, lleus, Intensive care, Motility disorder, Pseudo-obstruction.

Indian Journal of Critical Care Medicine (2020): 10.5005/jp-journals-10071-23614
\end{abstract}

\section{INTRODUCTION}

It is not uncommon to encounter gastrointestinal (GI) problems in intensive care unit (ICU) due to the advances in surgery and critical care. The incidence of GI motility disorders approximates $50-80 \%$ in the critically ill patients, and these substantially increase the mortality risk. An association between $\mathrm{Gl}$ motility disorders and increased incidence of infection, duration of mechanical ventilation, total length of hospital stay, and increased mortality has been reported in various studies. ${ }^{1}$ These pose a major hurdle in early, optimal enteral nutrition (EN) delivery, necessitating transient cessation of EN. ${ }^{2}$ The critical illness itself, head injury, burns, certain drugs, or surgical interventions can trigger variety of GI problems, including the inability to enterally feed the patients, ileus, diarrhea, and bacterial translocation. ${ }^{3}$

The purpose of this review is to focus on the common gastrointestinal tract (GIT) motility disturbances relevant in the ICU which includes gastroparesis, ileus, and acute colonic pseudoobstruction (ACPO/Ogilvie's Syndrome).

\section{Gastrointestinal Tract Motility- Normal Physiology}

By virtue of its motor function, GIT allows to mix and propel the food particles at a rate that facilitates nutrient absorption, and peristalsis so that the proximal intestine is cleared of residual food and bacteria, which ultimately leads to formation and movement of the stool. ${ }^{2,4}$ The central (CNS), autonomic (ANS), and enteric nervous systems (ENS) regulate the motility of gut, GI peptides, neurotransmitters, and hormones being the modulators. Myenteric and submucosal plexuses compose the ENS which is situated within the wall of GIT. It can function independent of the CNS. ${ }^{4}$ The migrating motor complex (MMC), which is controlled by ENS, is responsible for the motor activity of gut. Interdigestive (fasting) and digestive are the two motility patterns that have been identified and they consist of distinct phases. ${ }^{4}$

\section{Gastrointestinal Tract Motility-}

\section{Abnormal Physiology}

It is not unusual for the critically ill patients to be having GI motility disturbances, and these can involve the GIT either in parts, for
${ }^{1}$ Institute of Critical Care and Anesthesia, Medanta-The Medicity, Gurugram, Haryana, India

${ }^{2}$ Department of Critical Care Medicine, Medanta-The Medicity, Gurugram, Haryana, India

Corresponding Author: Deepak Govil, Institute of Critical Care and Anesthesia, Medanta-The Medicity, Gurugram, Haryana, India, Phone: +91-11-26692531, e-mail: drdeepak_govil@yahoo.co.in

How to cite this article: Govil D, Pal D. Gastrointestinal Motility Disorders in Critically III. Indian J Crit Care Med 2020;24(Suppl 4): S179-S182.

Source of support: Nil

Conflict of interest: None

example, the stomach, small intestine or colon, or in entirety. The etiology in critically ill is multifactorial and is precipitated by factors such as shock, multiple organ dysfunction syndrome, sepsis, trauma, brain injury, surgery, drugs (e.g., catecholamines, analgo-sedation), dyselectrolytemia, hyperglycemia, hypoxia, ischemia, dysregulation of gut hormones, and intra-abdominal compartment syndrome.

Disordered gut motility leads to small intestinal bacterial overgrowth, increased mucosal permeability, and bacterial translocation, which, in turn can cause diarrhea, decreased intravascular volume, the systemic inflammatory response syndrome (SIRS), sepsis, shock, and multiple organ dysfunction. It can also decrease the absorption and efficacy of nutrition and medications. Gastroparesis with high gastric residual volume (GRV) could be a factor responsible for aspiration pneumonia increasing the duration of hospital stay, morbidity and mortality. ${ }^{2,4}$

\section{Esophageal Motility Disorder Gastroesophageal Reflux Disease in Intensive Care Unit}

It is physiologic to have gastroesophageal reflux (GER) but a more frequent reflux or reflux causing esophageal mucosal injury is pathological. As per Montreal global consensus, gastroesophageal reflux disease (GERD) is a condition consisting of symptoms caused by reflux of gastric contents. ${ }^{1}$ 
In critical illness, all mechanisms that prevent esophageal epithelial injury due to gastric contents are disturbed.

A consistently decreased resting lower esophageal sphincter (LES) pressure found in these patients, along with increased intraabdominal pressure, is accountable for approximately $55 \%$ of the reflux episodes as shown by Nind et al. ${ }^{5}$ Also, LES relaxation could occur as a side effect of certain drugs whose use is relatively common in the ICU, for example, opioids, nitrates, calcium channel blockers, and barbiturates.

Another important factor responsible for increased risk of reflux in critical patients is supine position due to the lack of assistance from gravity. This is the reason why head end elevation is recommended to help clear the reflux. Many elements like presence of endotracheal tube, increase in intra-abdominal pressure, and medications that decrease gastric emptying, contribute to increase in frequency of transient LES relaxations.

Esophageal peristalsis is also jeopardized in patients on mechanical ventilation support. A common procedure in ICU patients, nasogastric intubation, and impaired salivary clearance are other factors implicated in GERD. ${ }^{1,6}$

There are various diagnostic tests suggested for esophageal dysmotility assessment, such as liquid-esophageal manometry, high-frequency endoluminal ultrasound, multichannel intraluminal impedance, and high-resolution manometry but are of limited use in ICU patients. ${ }^{6}$

\section{Gastric Motility Disorder}

\section{Gastroparesis in Intensive Care Unit}

Gastroparesis is a disorder of movement of stomach, wherein the gastric emptying (GE) is slow. The incidence of gastroparesis approximates $4-5 \%$ in general, while in critical care setting its prevalence is estimated to be even higher. ${ }^{1}$ With increasing severity of illness, the chances for delayed GE are increased, making it a common occurrence in ICU.,

Major pathophysiological mechanisms for delayed GE in these patients includes motor dysfunction in gut, lack of coordination between the fundic and pyloric regions of stomach, feedback inhibition from the adjoining small intestine, or a mix of any of these. ${ }^{6}$

The predisposing factors for gastroparesis includes comorbidities and chronic conditions such as diabetes mellitus, pancreatitis, obesity, elderly, hepatic cirrhosis, or acute conditions such as polytrauma, head injury, burns, sepsis, stomach cancer and surgery. It has been reported that hyperglycemia and dyselectrolytemia can cause a slowing of motility in the stomach, and proximal intestine. ${ }^{1,6}$

The various direct and indirect methods to assess gastric dysmotility in critical illness include GRV measurement, paracetamol absorption test, breath tests using isotope (e.g., $\left.{ }^{13} \mathrm{CO}_{2}\right)$, carbohydrate absorption, ultrasound, magnetic resonance imaging, scintigraphy, and wireless motility capsule. ${ }^{6}$ In ICU, in patients who are being fed enterally, use of intermittent measurement of GRVs is common to assess the GE and feeding tolerance, however, there is not enough evidence to support its utility. SCCM/ASPEN currently suggests the elimination of GRVs, for monitoring feed tolerance, completely from routine care. ${ }^{7}$ It is suggested to individualize monitoring GRVs as per the clinical setting, for example, postoperative abdominal surgery. As per the guidelines, it is suggested not to withhold enteral feeding for GRV $<500 \mathrm{~mL}$, unless patient shows any clinical signs of intolerance. $^{7}$

\section{Ileus}

lleus is described as lack of normal physiological intestinal motility, without any mechanical obstruction, resulting in inability to propel its contents further in the $\mathrm{GIT}^{8}{ }^{8}$ In critical illness, there is stimulation of sympathetic pathway with release of inhibitory inflammatory mediators which leads to hypomotility of the GIT. The etiology of ileus in the critical care setting is multifactorial, the usual etiologies being sepsis, inflammatory conditions, certain drugs, metabolic disturbances (Table 1). Of all the causes of ileus in ICU, postoperative ileus is the most common. Intestinal stasis is associated with bacterial overgrowth and translocation, SIRS, sepsis, and multiorgan dysfunction.

Ileus may present as inability to tolerate feeds, nausea, vomiting, lack of flatus or feces, abdominal distention, increased GRV, or absent bowel movements. Ileus leads to intestinal dilatation which further leads to increased intra-abdominal pressure. This can result in increased GRVs, enhanced risk of aspiration, and prolonged duration of mechanical ventilation. Plain X-ray abdomen shows the presence of gas distension of bowel loops and air fluid levels within them.

\section{Colonic Dysmotility in Intensive Care UNIT}

\section{Acute Colonic Pseudo-obstruction}

Acute colonic pseudo-obstruction (ACPO), also known as Ogilvie's syndrome, is an acute colonic dilatation without any mechanical obstruction, and is potentially fatal. ${ }^{8}$ The pathophysiology of ACPO

Table 1: Etiology of lleus in intensive care unit ${ }^{1}$

\begin{tabular}{|c|c|c|}
\hline Etiology & Conditions & Pathophysiology \\
\hline Inflammatory & $\begin{array}{l}\text { Sepsis, postop- } \\
\text { erative, pancreatitis, } \\
\text { cholecystitis, perito- } \\
\text { nitis, burns }\end{array}$ & $\begin{array}{l}\text { Release of nitric oxide, } \\
\text { prostaglandins, inflamma- } \\
\text { tory cytokines, vasoactive } \\
\text { peptide }\end{array}$ \\
\hline \multirow[t]{3}{*}{ Drugs } & $\begin{array}{l}\text { Opioids, anticholiner- } \\
\text { gics, calcium channel } \\
\text { blockers, vasopres- } \\
\text { sors, excessive IV } \\
\text { fluid }\end{array}$ & $\begin{array}{l}\text { Opioids act on the mu } \\
\text { and delta opioid receptors } \\
\text { located on the smooth } \\
\text { muscle of the gut wall } \rightarrow \\
\text { decreased levels of c AMP } \\
\text { and calcium } \rightarrow \text { decreased } \\
\text { excitatory neurotransmit- } \\
\text { ter release } \rightarrow \text { decreased } \\
\text { peristalsis. }\end{array}$ \\
\hline & & $\begin{array}{l}\text { Vasopressors decrease } \\
\text { perfusion to the GIT. }\end{array}$ \\
\hline & & $\begin{array}{l}\text { Excess IV fluids cause } \\
\text { intestinal wall edema }\end{array}$ \\
\hline \multirow[t]{2}{*}{ Metabolic } & $\begin{array}{l}\text { Hyperglycemia, } \\
\text { Hypokalemia, } \\
\text { hypomagnesemia, } \\
\text { acidosis, hypoxia, } \\
\text { hypovolemia }\end{array}$ & $\begin{array}{l}\text { Impaired gut motility due } \\
\text { to altered nerve conduc- } \\
\text { tion. }\end{array}$ \\
\hline & & $\begin{array}{l}\text { Hypovolemia leads to } \\
\text { decreased gut perfusion }\end{array}$ \\
\hline Critical illness & $\begin{array}{l}\text { Sepsis, trauma, renal } \\
\text { failure, mechanical } \\
\text { ventilation, multior- } \\
\text { gan failure }\end{array}$ & $\begin{array}{l}\text { Activation of inhibitory } \\
\text { sympathetic input and the } \\
\text { release of inflammatory } \\
\text { mediators that inhibit gut } \\
\text { motility. }\end{array}$ \\
\hline
\end{tabular}


is unclear, although the prevailing hypothesis is that it is the result of an increased sympathetic and decreased parasympathetic activity in the colon leading to lack of peristalsis. There are various medical and surgical conditions, drugs like narcotics and anticholinergics, sepsis, and cytomegalovirus infection, which have been found to be associated with ACPO. These also include mechanical ventilation, neurological disorders like parkinsonism, stroke, and multiple sclerosis, dyselectrolytemia (hypokalemia, hyponatremia, hypocalcemia/hypercalcemia), diabetes, hypothyroidism, burns/ trauma, pancreatitis, myocardial infarction, pneumonia, etc. ${ }^{1}$

Abdominal distension is the chief presenting symptom and is usually associated with pain abdomen, nausea, and vomiting. In up to $40 \%$ of patients, the passage of flatus and feces remains undisturbed, and bowel sounds are usually present. Presence of fever, peritoneal signs, including tenderness, guarding, rigidity, and leukocytosis, are usually suggestive of its complications, that is, bowel ischemia or perforation. ${ }^{9}$

Typically, colonic dilation is seen on a plain X-ray. ${ }^{6}$ Abdominal computed tomography scan is usually done to rule out mechanical obstruction. ${ }^{1}$ On imaging, haustrations are maintained in ACPO, that differentiates it from toxic mega-colon and acute colon obstruction. ${ }^{1}$ It more commonly involves the cecum and the ascending and transverse colon, although left colon may also be affected. ${ }^{1,6}$ Cecal dilation with diameter $>12 \mathrm{~cm}$ is predictive of high risk of perforation. ${ }^{6}$

\section{Management}

\section{General Therapeutic Measures for Gastrointestinal Motility Disorders}

- Intravenous fluids-Tailored fluid administration should be done in postoperative or critically ill patients, so as to avoid either hypo- or hyper-volemia, as even excess fluids can cause bowel wall edema and are associated with poorer outcome. ${ }^{9}$

- Correction of dyelectrolytemia ${ }^{6,9}$

- Control of blood sugars/hyperglycemia-high blood sugars have been associated with delayed gastric emptying, and a negative effect on antral motor activity and duodenum and jejunum motility.

- Early enteral feeding - It is recommended to initiate EN early in the critical care setting, that is, within 24-48 hours, as it is important in maintaining structural and functional intestinal integrity. ${ }^{7,9}$ It is recommended to divert the level of infusion further in the GIT in those patients who are at increased risk of aspiration or have shown signs of intolerance to gastric feeding. A close watch should be observed for any signs of feed intolerance as early indicators of bowel ischemia (e.g., abdominal distension, high gastric aspirate, inability to pass flatus or stool, sluggish bowel sounds, metabolic/lactic acidosis) in patients who are on increasing vasopressor support and receiving EN, and enteral feed should be withheld until stabilization. ${ }^{7}$ Avoid use of nasogastric (NG) tubes where patient can accept oral feed so as to decrease risk of GER, risk of esophageal stricture, and other complications such as sinusitis and sore throat. The incidence of reflux has been found to be less with percutaneous endoscopic gastric (PEG) feeding when compared to NG feeding in both ventilated and non-ventilated patients. ${ }^{1}$

- Judicious use of catecholamines and medications used for analgo-sedation $^{9}$

\section{Specific Therapeutic Measures}

Treatment of Gastroparesis

Pharmacological approach includes use of: ${ }^{1,6}$

- Motilin receptor agonists-Erythromycin, a prokinetic, is more effective when given intravenously. Others include atilmotin, mitemcinal, and ghrelin.

- Dopamine receptor antagonists-Metoclopramide acts by antagonizing central and peripheral dopamine D2 receptor, and stimulating 5-hydroxytryptamine $(5-\mathrm{HT})$ receptors. This causes release of acetylcholine in the gut wall. It has a potential for neurological side effects, such as dystonia and Parkinson's syndrome. On the other hand, domperidone, available only as oral formulation, acts by antagonizing peripheral dopamine $D 2$ receptor that accelerates GE by inhibiting fundic relaxation. Sulpiride is a dopamine antagonist. Itopride is a D2 antagonist with anti-acetylcholinesterase activity.

- 5-HT4 agonists-Cisapride is a 5-HT 4 agonist but has been withdrawn from market due to its cardiac side effects. Because of the controversial evidence on GE and its cardiovascular side effects, tegaserod use is not approved for gastroparesis.

\section{Treatment of lleus}

Pharmacotherapy for ileus includes: ${ }^{1,6}$

- Dopamine receptor antagonists-Use of metoclopramide for ileus is controversial and is associated with neurological side effects. With domperidone extrapyramidal side effects are very rare. It can cause hyperprolactinemia and QT interval prolongation.

- Motilin receptor agonist-Erythromycin increases GE by stimulating motilin, with a limited utility in ileus. Also, it can cause QT interval prolongation and increase in transaminases. Tachyphylaxis to erythromycin monotherapy develops fast.

- 5-HT4 agonist-Cisapride, mosapride, and prucalopride act by increasing cholinergic activity. They have direct effect on colon, and are also associated with cardiovascular side effects.

- Mu opioid receptor antagonist-Various studies have reported methylnaltrexone to shorten the time to first bowel movement postoperatively.

- Osmotic laxatives-Lactulose and polyethylene glycol have been used in chronically constipated patients in ICU, and their efficacy is at par in facilitating defecation.

- Chloride channel activator-Lubiprostone is used mainly in patients with opioid-induced ileus and chronic constipation associated with Parkinsons disease.

\section{Treatment of Acute Colonic Pseudo-obstruction}

It consists of pharmocological, endoscopic and surgical approaches. ${ }^{1}$

\section{Pharmacological Agents}

- Neostigmine-It is a short-acting acetylcholinesterase inhibitor that by potentiating action of acetylcholine on muscarinic receptors on the GIT improves the motility. Administered IV as $2 \mathrm{mg}$ to $2.5 \mathrm{mg}$ over $3-5$ minutes, it is the first choice for treatment of pseudo-obstruction. Its adverse effects include arrhythmias, particularly, bradycardia and atrioventricular block, and is contraindicated in patients having arrhythmias, bronchospasm, or renal failure. 
- Pyridostigmine-It is a long-acting acetylcholinesterase inhibitor. Its use has been described in small studies in cases of recurrent $A C P O$, with $10 \mathrm{mg}$ given per-oral orally twice daily as the initial dose.

- Opioid receptor antagonists-Methylnaltrexone and alvimopan can be used in opioid-induced ileus. Their use in ACPO is yet to be proven.

- Polyethylene glycol-It is an osmotic laxative, the use of which has shown a decreased recurrence of ACPO in small prospective randomized controlled trials.

Endoscopic Management: It is indicated for refractory ACPO when the patient does not respond to the pharmacological therapy. The risk associated is that of perforation, and the recurrence rate approximates around $40 \%$. Colonoscopic decompression with or without placement of decompression tube can be done. Percutaneous endoscopic cecostomy (PEC) is also an option but complication rate is higher with this, which includes perforation, peritonitis, bleeding, and the migration of the PEC tube. ${ }^{1,8}$

Surgical Management: Surgical interventions, including cecostomy, colostomy or colectomy, are reserved for patients with refractory ACPO leading to abdominal compartment syndrome or other complications. ${ }^{1}$

\section{CONCLUSION}

GI motility disorders and feeding intolerance are frequently encountered in ICU and cause a significant increase in morbidity and mortality. They can result in malnutrition, bacterial translocation, sepsis, and multiorgan dysfunction. It is important to recognize the location and type of the motility disorder so that an appropriate therapy can be chosen. There is a need for development of newer methods for assessment of Gl motility in critically ill patients for their appropriate and early management and prevention of complications.

\section{References}

1. Aderinto-Adike AO, Quigley EMM. Gastrointestinal motility problems in critical care: a clinical perspective. J Dig Dis 2014;15(7):335-344. DOI: 10.1111/1751-2980.12147.

2. Retief I. The management of motility disorders in critical illness. S Afr J Clin Nutr 2011;24(3):S15-S18. DOI: 10.1080/16070658.2011. 11734374.

3. Fruhwald S, Kainz J. Effect of ICU interventions on gastrointestinal motility. Curr Opin Crit Care 2010;16(2):159-164. DOI: 10.1097/ MCC.0b013e3283356679.

4. Ukleja A. Altered GI motility in critically ill patients: current understanding of pathophysiology, clinical impact, and diagnostic approach. Nutr Clin Pract 2010;25(1):16-25. DOI: 10.1177/0884533609357568.

5. Nind G, Chen WH, Protheroe R, Iwakiri K, Fraser R, Young R, et al. Mechanisms of gastroesophageal reflux in critically ill mechanically ventilated patients. Gastroenterology 2005;128(3):600-606. DOI: 10.1053/j.gastro.2004.12.034.

6. Ladopoulos T, Giannaki M, Alexopoulou C, Proklou A, Pediaditis E, Kondili E. Gastrointestinal dysmotility in critically ill patients. Ann Gastroenterol 2018;31:273-281. DOI: 10.20524/aog.2018.0250.

7. Taylor BE, McClave SA, Martindale RG, Warren MM, Johnson DR, Braunschweig C, et al. Guidelines for the provision and assessment of nutrition support therapy in the adult critically ill patient: Society of Critical Care Medicine (SCCM) and American Society for Parenteral and Enteral Nutrition (A.S.P.E.N.). Crit Care Med 2016;44(2):390-438. DOI: $10.1097 / C C M .0000000000001525$.

8. Vazquez-Sandoval A, Ghamande S, Surani S. Critically ill patients and gut motility: are we addressing it? World J Gastrointest Pharmacol Ther 2017;8(3):174-179. DOI: 10.4292/wjgpt.v8.i3.174.

9. Fruhwald $\mathrm{S}$, Holzer $\mathrm{P}$, Metzler H. Gastrointestinal motility in acute illness. Wien Klin Wochenschr 2008;120(1-2):6-17. DOI: 10.1007/ s00508-007-0920-2. 